\title{
Correction to: Sparse subsets of the natural numbers and Euler's totient function
}

\author{
MITHUN KUMAR DAS, PRAMOD EYYUNNI \\ and BHUWANESH RAO PATIL*
}

Harish-Chandra Research Institute, HBNI, Chhatnag Road, Jhunsi, Allahabad 211 019, India *Corresponding author.

E-mail: das.mithun3@gmail.com; pramodeyy@gmail.com; bhuwanesh1989@gmail.com

MS received 25 November 2019; accepted 8 January 2020; published online 21 April 2020

\section{Correction to: Proc. Indian Acad. Sci. (Math. Sci.) (2019) 129:84 https://doi.org/10.1007/s12044-019-0512-x}

Jean-Marc Deshouillers has noticed an error in the proof of Proposition 20 in the article [1]. More precisely, the 10th and 11th lines of the proof of Lemma 18 on page 7 in the article [1], which is displayed below, are not correct:

$$
\begin{aligned}
\frac{\left|V \cap F_{n}\right|}{\left|F_{n}\right|} & =\frac{\left|V \cap\left[1, x_{n}\left(1+\alpha_{n}\right)\right]\right|-\left|V \cap\left[1, x_{n}\right]\right|}{\left|F_{n}\right|} \\
& \leq \frac{1}{\alpha_{n} \log x_{n}}\left(\left(1+\alpha_{n}\right) \exp \left(\left(1+\alpha_{n}\right)^{2} z_{n}\right)-\exp \left(z_{n}\right)\right) .
\end{aligned}
$$

Therefore, the proof given in the paper does not guarantee the conclusion of Lemma 18. Since the proof of Proposition 20 uses this lemma, its proof no longer holds. In effect of this, there is no requirement of Lemmas 16 and 17 because these lemmas are only used for the proof of Proposition 20. The corrected sentences in the article [1] are given below:

1. Last sentence of the paragraph just before Theorem 3 on page 2:

The first theorem in this paper measures the densities of sets $N_{1}, N_{2}, N_{3}$ etc.

2. First sentence under section "Theorem 3"(page 2):

(i) The Banach density of $N_{1}$ is zero.

3. First sentence under section "2.1 Proof of Theorem" (page 6):

We now evaluate the densities of the sets $N_{1}, N_{2}, N_{3}$ and more generally, for sets of the form $f(V)$, where $f: V \rightarrow \mathbb{N}$ is an injective map such that $f(m) \in \phi^{-1}(m)$.

4. The following results along with their proofs should be deleted:

(a) Lemma 16 on page 6 ,

(b) Lemma 17 on page 6-7,

The original article can be found online at https://doi.org/10.1007/s12044-019-0512-x. 
(c) Lemma 18 on page $7-8$,

(d) Proposition 20 on page 8.

5. The following line on page 9 should be deleted.

"Next, we proceed to prove that the Banach density of $N_{1}$ is zero."

6. Third sentence in the proof of Corollary 22 (page 9):

Since $\bar{d}_{F_{n}}(V)=0$, by Lemma 19 , it follows that $\bar{d}_{F_{n}}(f(V))=0$, by applying Lemma 21.

7. First sentence on page 10:

Hence, the proof of Theorem 3 is complete by collecting Proposition 23 and Corollary 22.

8. The paragraph between Question 1 and Question 2 in Section 5 on page 25 should be replaced by the following:

"In relation to densities of $N_{2}$ and $N_{3}$, we ask:".

These corrections do not affect any other result in article [1].

\section{Acknowledgements}

The authors would like to thank Prof. Jean-Marc Deshouillers for his observations.

\section{Reference}

[1] Das M K, Pramod E and Bhuwanesh R P, Sparse subsets of the natural numbers and Euler's totient function, Proc. Indian Acad. Sci. (Math. Sci.) 129 (2019) 84. https://doi.org/10.1007/ s12044-019-0512-x 